\title{
Abstractions
}

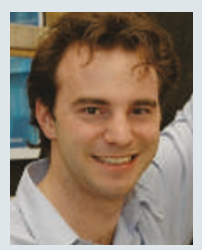

\section{PROJECT LEADER}

Light falling on a tiny mirror exerts a pressure, causing the mirror to move and potentially revealing its quantummechanical behaviour. But at room temperature the effects are masked because the mirror is in a constant state of thermal agitation. Markus Aspelmeyer at the Institute for Quantum Optics and Quantum Information in Vienna, Austria, and his team have found a way to cool the micromirror using radiation pressure, and thus damp down this random agitation. Furthermore, their system can selfregulate its own cooling, removing the need for any active feedback loops. Their work on page 67 creates an important avenue for physicists to combine micromechanical motion and radiation to improve the sensitivity of high-precision measures - or one day even demonstrate quantum behaviour.

\section{Why has no one achieved self-cooling by radiation pressure in a micromechanical system until now?}

There have been attempts over the past 5 to 10 years, but no one had a micromirror that was lightweight enough, or highly reflecting. We were lucky to collaborate with guys from the United States and Austria who could work with our initially rough ideas for such high-sensitivity systems - which are only now possible because of nano- and microfabrication techniques.

\section{What was the key breakthrough?} It was really a series of minor breakthroughs, but we did celebrate at our first successful attempt to fabricate the mirror used in the experiment. Our celebratory, now empty, bottle of champagne has a plot of our first characterization of the mirror on it.

The paper was put on hold while control experiments were reworked - during the World Cup no less. What happened?

We were ambitiously trying to provide the first full quantum description, rather than follow previous classical-physics attempts, so we had to be extremely careful. At one point we realized there seemed to be a factor of two missing when we compared our findings with results in the classical literature. It turned out that our original data were correct, and luckily we were able to settle all experimental and theoretical issues before the World Cup semi-finals.

\section{What's the next step?}

Classical physics offers no means to describe the quantum ground state, or lowest possible energy state. By continuing the work described in this paper and eliminating the current mirror imperfections, we will get there straight away.
MAKING THE PAPER

\section{Eberhard Fetz}

\section{Computer-chip implants could help people with brain damage.}

As part of a neuronal relay race, electrical signals travel from the brain to the muscles that control movement. Break any neuronal connection in the chain - through injury or disease - and the control may be lost.

Eberhard Fetz and his colleagues at the University of Washington, Seattle, attempted to use a small computer chip to replace these lost connections. But in experiments described on page 56 , they found that their device could also serve to strengthen and rewire existing ones.

The collaboration between Fetz, Andrew Jackson and Jaideep Mavoori began in 2003, when Mavoori, an electrical engineer, was developing a computer chip that could be implanted in moths to record and stimulate neuronal activities as they flew.

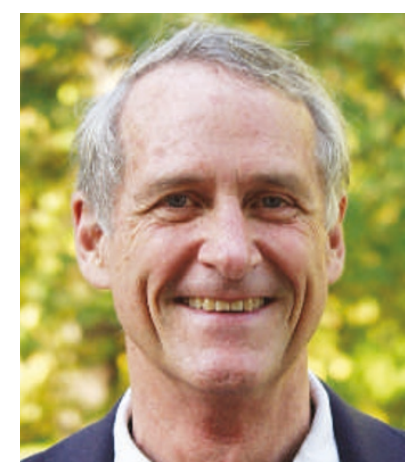

small, but possess enough battery power to run for several days, and enough computational power to record signals and analyse the data in real time, according to Mavoori.

The chip also had to be sufficiently stable to function in living animals. "Monkeys do not sit very still. They don't seem to care if you are trying to do science," laughs Fetz, who worried that such movements would create too much background noise. But once implanted, the chip worked. "I remember the first time we downloaded the data from the chip and got a glimpse of a day in the life of a brain cell," recalls Jackson. "It was very exciting."

They next asked whether the microchip was having any effect on brain functions. At the start of the experiment, they used an external stimulator to test different sites in the monkeys' motor cortex. As expected, stimulation of site A caused wrist muscles to twitch in one direction, and stimulation of site B caused twitching in another. Then the monkeys were left to go about their business with the chip on their brain, where it recorded and transmitted signals. After a couple of days, "We heard about the work and thought something that small should be easy to implant into a monkey's brain," recalls Fetz. That insight turned out to be correct, although implementing it was not easy.

The team spent more than a year redesigning the chip so that it could be programmed to record signals from neurons firing in an area of the brain's motor cortex responsible for moving certain wrist muscles in one direction (site A). The chip, which was positioned on top of the brain, then delivered these signals a few milliseconds later to a nearby site responsible for controlling a different set of wrist muscles (site B). The chip made the recordings and stimulations through two implanted electrodes, thus providing a constant connection between the two sites in the brain. The device had to be the researchers once again tested different areas of the brain with the external stimulator. They found that now stimulation of site A was causing the wrist to move in the same direction as stimulation of site B. Thus the artificial connection between A and B had changed A's function to be more like B's.

"I was rather surprised when we got the result," says Jackson. "We saw a change of output from a whole region of the cortex and this caused a lasting change in the movement produced by stimulation." This change in function, called plasticity, is thought to occur normally during learning and memory. The researchers speculate that in people who have suffered brain damage, the chip could be used to help reorganize information flow and boost the functioning of spared neurons.

\section{KEY COLLABORATOR}

In lieu of exact species counts,

conservation strategies typically rely on the assumption that geographic diversity patterns are the same among all

taxonomic groups. A 16-strong team of young conservation biologists, many already friends, decided to test that assumption.

Former housemates and $\mathrm{PhD}$ labmates Richard Grenyer and David Orme kept in touch while doing their respective postdocs. Grenyer was working on a massive mammal database at the University of Virginia in Charlottesville, while Orme, based at Imperial College London, UK, focused his attention on a global distribution of birds. Inspired by the compatible data sets, they combined them with an amphibian database to form a huge inventory of more than 19,000 species. On page 93 , the team reports that whereas the distribution of overall species richness is similar among the three groups, there is little similarity in the distribution patterns of rare and threatened species.

"All of the data coming online at the same time was critical," says Grenyer, now a researcher at the Royal Botanic Gardens in Kew, London. But the key to this probably controversial work, he adds, was working with friends. "If you're working with people you don't know, or who are senior to you, your creativity may be constrained. You don't take risks," he says. 